\title{
La gestion de l'occlusion intestinale chez les patientes atteintes du cancer de l'ovaire au moyen d'une sonde de gastrostomie endoscopique percutanée (GEP)
}

\section{Abrégé}

On estime qu'en 2002, 2500 femmes du Canada ont été diagnostiquées du cancer de l'ovaire et que 1500 en sont décédées (Institut national du cancer du Canada, 2002). Jusqu'à $42 \%$ des femmes en phase palliative sont atteintes d'une occlusion intestinale maligne. Pour contrôler cette dernière, les options incluent un traitement médical, la chirurgie et/ou la pose d'une sonde de gastrostomie endoscopique percutanée (GEP).

L'objectif de cette étude d'amélioration de la qualité consistait à 1) examiner si la pose d'une sonde de PEG s'était soldée par un soulagement efficace, et 2) dégager des possibilités d'amélioration de la qualité des soins infirmiers prodigués. Une étude rétrospective des dossiers de 24 patientes a révélé que $75 \%$ d'entre elles n'avaient ni nausée ni vomissements au moment du congé; que $92 \%$ reprenaient un régime de liquides clairs; que $83 \%$ recevaient leur congé de l'unité de soins intensifs; et enfin, que $70 \%$ ne subissaient pas de réadmission. Une sonde de GEP peut soulager efficacement les symptômes des femmes présentant une occlusion intestinale non opérable associée à un cancer de l'ovaire avancélrécurrent. Des possibilités d'amélioration des soins sont présentées.

Parmi les cancers gynécologiques, le cancer de l'ovaire est celui qui entraîne le plus souvent la mort; il est de plus la cinquième cause de décès par cancer chez les femmes au Canada et aux États-Unis. On estime qu'en 2002, 2500 femmes du Canada ont été diagnostiquées du cancer de l'ovaire et que 1500 en sont décédées (Institut national du cancer du Canada, 2002). Malgré une amélioration considérable des taux relatifs de survie à cinq ans entre le milieu des années 70 et celui des années 90, le taux de survie reste tristement bas: $44 \%$ pour l'ensemble des stades. Le cancer de l'ovaire étant habituellement asymptomatique dans ses étapes précoces, seules $25 \%$ des patientes sont diagnostiquées d'un cancer localisé. Les femmes diagnostiquées en stade III et IV ont un taux de survie à cinq ans allant de $10 \%$ à $30 \%$ (DiScia et Creasman, 2002).

Les écrits de recherche indiquent que de 5,5 à $25 \%$ des femmes atteintes du cancer de l'ovaire souffrent tôt ou tard d'une occlusion intestinale maligne et que jusqu'à $42 \%$ des femmes en phase terminale en souffrent (Ripomonti, 1994). L'occlusion intestinale est souvent le premier signe d'un cancer de l'ovaire récurrent. Dans de tels cas, l'occlusion intestinale est associée à un pronostic sombre et signale la proximité de la phase terminale (Beattie, Leonard et Smyth, 1989). L'objectif du traitement est de soulager les symptômes et de rehausser la qualité de vie, puisque le cancer ne répondra sans doute plus à la thérapie et que l'occlusion ne se résorbera probablement pas.

Dans les cas de cancer de l'ovaire, la pathophysiologie de l'occlusion est associée à la compression extrinsèque de l'intestin et à la carcinomatose de l'iléus, et elle se caractérise par une perturbation de la motilité intestinale (Cain, Wenzel, Monk et Cella, 1998). Le lieu d'occlusion le plus courant est l'intestin grêle (Feuer, Broadley et Barton, 2000).

En matière de gestion de l'occlusion intestinale, les options incluent la laparotomie avec pontage chirurgical, la pharmacothérapie, la chimiothérapie et le drainage par gastrostomie endoscopique percutanée (GEP) ou par sonde nasogastrique (Jacobs et coll., 2002). Cependant, la gestion optimale de l'occlusion intestinale associée au cancer de l'ovaire récurrent demeure une question controversée.

En matière de gestion de l'occlusion intestinale, la chirurgie continue de présenter le meilleur résultat à long terme en matière de survie, mais il convient de procéder avec extrême circonspection afin de déterminer quelles patientes pourraient bénéficier de la chirurgie (Gadducci et coll., 1998; Jong, Sturgeon et Jamieson, 1995; Ripomonti, 1994; van Ooijen, van der Burg, Planting, Siersema et Wiggers, 1993). Le système de notation mis au point par Krebs et Goplerud propose des critères d'admissibilité fiables (mentionnés dans Gadducci et coll.). Ce système est fondé sur les variables suivantes: l'âge de la patiente, son état nutritionnel, l'état de ses tumeurs, le nombre d'ascites et ses antécédents en matière de traitements de chimiothérapie et de radiothérapie. Jong et collègues ont réalisé un examen rétrospectif des dossiers qui a révélé que le traitement palliatif avait réussi chez $51 \%$ des patientes qui ne présentaient aucun des quatre facteurs pronostiques suivants: la présence d'une masse palpable au niveau de l'abdomen et du bassin, la présence d'ascites dépassant 3 litres, des sites d'occlusion multiples et une perte de poids préopératoire de plus de $9 \mathrm{~kg}$. On utilise des critères semblables afin d'identifier les patientes à qui il convenait de proposer la possibilité d'une opération chirurgicale dans notre centre. L'emploi de tels critères peut éventuellement éviter les interventions inutiles et réduire le taux de morbidité parmi les patientes.

En plus de la chirurgie, la chimiothérapie constitue une option thérapeutique intéressante lorsqu'on souhaite contrôler l'évolution du cancer et soulager les symptômes de l'occlusion intestinale. Dans leur étude, Abu-Rustum, Barakat, Venkatramann et Spriggs (1997) ont conclu que la chimiothérapie était inefficace dans la restauration des fonctions intestinales chez les femmes ayant déjà subi des traitements intensifs de chimiothérapie pour leur cancer de l'ovaire récurrent. Ils recommandaient que ce traitement ne soit offert qu'aux patientes n'ayant jamais reçu de traitements de chimiothérapie.

Dans les cas où la chirurgie et la chimiothérapie ne sont pas des options thérapeutiques viables, il revient aux professionnels de la santé d'aider les patientes et leur famille au niveau de la prise de décisions quant au contrôle optimal des symptômes de l'occlusion intestinale et à l'optimisation de leur qualité de vie.

La littérature décrit également le contrôle, au moyen d'un traitement pharmacologique, des symptômes de l'occlusion intestinale chez les femmes atteintes d'un cancer de l'ovaire en phase terminale (Baumrucker, 1998; Gadducci et coll., 1998; Mangili et coll., 1996; Ripomonti, 1994). Cette approche a été adoptée avec succès dans divers hôpitaux. Pour leur part, Fainsigner, Spachynski, Hanson et Bruera (1994) ont réalisé un examen rétrospectif des dossiers des

Lynne Jolicoeur, RN, BScN, CON(C), Hôpital d'Ottawa, Campus Général, Ottawa, ON

Wylam Faught, MD, FRCSC, est Professeur et directeur, Department of Obstetrics and Gynecology, Chef, Women's Health Program, 205 CSC, Royal Alexandra Hospital, Edmonton, AB. 
patientes traitées dans leur unité de soins palliatifs où elles avaient été admises pour raison d'occlusion intestinale. Leur régime médicamenteux comprenait les produits suivants: le Dexamethasone, à une dose moyenne de $40 \mathrm{mg} /$ jour (de 8 à $60 \mathrm{mg}$ ); le Methoclopramide, $10 \mathrm{mg} / \mathrm{q} 4 \mathrm{~h}$, avec augmentation à une dose de 60 à $120 \mathrm{mg} / \mathrm{jour} \mathrm{chez}$ quatre des quinze patientes. Les autres médicaments employés incluaient le Dimenhydrate, le Haloperidol, le Domperidone et le Buscopan. La condition de $26 \%$ des patientes traitées dans leur unité de soins palliatifs était gérée au moyen d'une sonde de GEP. Les auteurs ont conclu que l'occlusion intestinale maligne peut être gérée avec succès en ayant très peu recours à la réhydratation par sonde nasogastrique ou par voie intraveineuse. La recherche décrit un grand nombre de protocoles différents, mais toutes les études sont d'accord sur un point: le régime doit être conçu sur mesure pour chaque patiente. (Baumrucker; Gadducci et coll.; Mangili et coll.; Ripomonti).

Certains chercheurs (p. ex. Khoo et coll., 1994; Mangili et coll., 1996; Mercadante, Spoldi, Caraceni, Maddaloni et Simonetti, 1993) ont trouvé que l'Octreotide était efficace dans le contrôle de la quantité de liquide gastrique produit et dans le soulagement des symptômes de l'occlusion intestinale. Soixante-deux pour cent des patientes participant à l'étude de Mangili et collègues ont pu retourner chez elles.

Des auteurs recommandent, dans les écrits, l'utilisation d'une sonde de gastrostomie endoscopique percutanée (GEP) comme moyen de gérer l'occlusion intestinale maligne lorsque la chirurgie n'est pas indiquée ou lorsqu'elle a échoué (Frankel Kelvin et Scagliola, 1998; van Ooijen et coll., 1993) ou lorsqu'une approche pharmacologique n'est pas efficace en milieu hospitalier (Baumarucker, 1998; Ripomonti, 1994). Les études rapportent que la sonde de GEP constitue une méthode de décompression gastrique à la fois plus économique et plus sécuritaire que la sonde nasogastrique et les autres sondes de gastrostomie (Adelson et Kasowitz, 1993; Marks, Perkal et Schwartz, 1993). Toutes les études disponibles concluent que la sonde de GEP est sécuritaire et qu'elle est associée à un taux de morbidité inférieur à celui qui correspond à l'utilisation de la sonde nasogastrique ou à la gastrostomie réalisée par laparotomie.

$\mathrm{Au}$ cours des trois dernières années, le traitement en vigueur dans notre centre pour les patientes souffrant d'occlusion intestinale due au cancer de l'ovaire récurrent a été le drainage par sonde de gastrostomie endoscopique percutanée (GEP) dont l'objectif est de maîtriser les symptômes de l'occlusion. Le traitement peut être subi soit au domicile soit à l'hôpital. La phase initiale de la thérapie est conservatrice: elle comprend l'aspiration nasogastrique, la réhydratation par voie intraveineuse, l'administration de corticostérö̈des et, dans certains cas, celle d'agents stimulant la motilité gastrique. Dans tous les cas, on envisage d'abord l'option du pontage chirurgical. Lorsque le chirurgien juge cette intervention non indiquée, on demande une consultation auprès du service de gastro-entérologie en vue d'insérer une sonde de GEP. L'insertion de la sonde se fait dans la salle de traitement endoscopique. On prépare la patiente en lui administrant les médicaments appropriés et en s'assurant que le site d'insertion ne présente aucun signe de métastase. Une fois la peau préparée, le chirurgien insère la sonde de GEP par la bouche de la patiente d'un seul geste régulier et la fait ressortir par la plaie abdominale. Cette technique est décrite dans l'étude de Marks et collègues (1993).

Une fois la patiente revenue dans l'unité, la sonde de GEP est reliée à un sac collecteur Foley pour le drainage par gravité. On bloque la sonde de GEP trente minutes avant les repas et la prise de médicaments par voie orale, ou aussi longtemps que la patiente ne ressent aucune nausée, ne vomit pas et/ou ne souffre pas de ballonnement abdominal. Dans les cas où le drainage par gravité est insuffisant pour contrôler la nausée et les vomissements, la sonde de GEP peut être reliée à un appareil à succion intermittente ou continue. Le régime alimentaire initial des patientes consiste en liquides clairs, puis on y ajoute d'autres sortes de liquides, des purées et finalement, des aliments à résidu faible, selon la tolérance. Au début, l'ingestion par voie orale est limitée aux liquides qui peuvent passer par la sonde de GEP. Une fois les symptômes un peu mieux maîtrisés, on bloque la sonde pendant des périodes de plus en plus prolongées. Si la patiente tolère bien le blocage de la sonde, on lui administre une alimentation à résidu faible. Dans le cas des patientes chez qui les fonctions intestinales reprennent, on garde la sonde de GEP bloquée et on la purge de trois à quatre fois par jour afin qu'elle conserve sa fonctionnalité. La sonde peut également servir à l'administration de médicaments par suspension orale. On n'enlève pas la sonde, puisque dans la plupart des cas, on peut s'attendre au retour des symptômes d'occlusion (Feuer et coll., 2000).

Étant donnée l'utilisation fréquente des sondes de GEP au sein de notre clinique pour soulager l'occlusion intestinale chez les femmes atteintes du cancer de l'ovaire, nous avons conçu une étude d'amélioration de la qualité à objectif double: (1) savoir si la sonde de GEP permet de contrôler les symptômes chez les patientes atteintes d'un cancer récurrent de l'ovaire ou du péritoine et souffrant d'occlusion intestinale, et (2) dégager des façons d'améliorer la qualité des soins infirmiers prodigués à ces femmes.

\section{Méthodologie}

Les auteurs de cet article ont réalisé un examen rétrospectif des dossiers de toutes les patientes souffrant d'occlusion intestinale qui ont été traitées à l'aide d'une sonde de GEP entre 1996 et 1999. À l'époque où nous avons débuté l'étude, il n'était pas nécessaire d'obtenir l'approbation du comité de déontologie pour réaliser des examens rétrospectifs des dossiers en vue de rehausser la qualité des interventions. À partir du système d'information sur les patientes du Service d'oncologie gynécologique, nous avons recueilli le dossier de 32 patientes traitées dans le centre. Huit des patientes ont été exclues d'emblée de l'étude, quatre d'entre elles parce que l'insertion de la sonde de GEP n'avait pas réussi en raison de la présence d'ascites et/ou de métastases péritonéales dans la région gastrique, et quatre autres parce que leur cancer ne touchait ni les ovaires ni le péritoine. L'information extraite incluait les données démographiques, les antécédents en matière de chirurgie et de chimiothérapie, les admissions passées pour raison d'occlusion intestinale, le lieu de l'occlusion, la durée du séjour, les symptômes lors du congé, le lieu de résidence après le congé, le régime alimentaire lors du congé, les autres méthodes employées pour gérer l'occlusion intestinale, le nombre de réadmissions après l'installation de la sonde de GEP, et le cas échéant, la date et le lieu de décès.

Toutes les patientes ont reçu un diagnostic d'occlusion intestinale après examen clinique et analyse radiologique. La condition des patientes était initialement gérée de façon conservatrice, tel que mentionné ci-dessus. Le cas particulier de chaque patiente avait été examiné afin de déterminer si une intervention par laparotomie était indiquée, mais aucune des patients n'en avait subi une. Après consultation auprès du service de gastroentérologie, les patientes avaient été munies d'une sonde de GEP dans le cadre de leur programme de soins palliatifs.

Les observations suivantes déterminaient si le contrôle des symptômes avait réussi: la disparition des symptômes de l'occlusion (nausée, vomissements et crampes/douleurs abdominales), la capacité de la patiente à reprendre au moins un régime de liquides clairs, l'absence de complications liées à la sonde de GEP, l'obtention du congé du service de soins intensifs et la non-réadmission à l'hôpital pour motif d'occlusion intestinale.

\section{Résultats}

Les 24 patientes incluses dans cette étude avaient reçu un diagnostic d'occlusion intestinale, et $88 \%$ d'entre elles $(n=21)$ avaient également été diagnostiquées d'une maladie récurrente ou évolutive. Trois patientes $(12 \%)$ avaient commencé à souffrir d'occlusion moins de trente jours après l'établissement de leur diagnostic primaire. Soixantedix-neuf pour cent des patientes $(n=19)$ avaient subi une intervention chirurgicale de réduction du volume tumoral, et $92 \%$ d'entre elles $(n=22)$ avaient reçu des traitements de chimiothérapie. Dans $79 \%$ des 
cas, l'occlusion se présentait au niveau de l'intestin grêle. Cinquante pour cent des patientes avaient déjà été admises une ou deux fois auparavant en raison d'une occlusion intestinale, et une autre avait déjà rapporté des symptômes précurseurs de l'occlusion intestinale.

En moyenne, l'insertion de la sonde de GEP a eu lieu 8,1 jours après l'admission, et le séjour durait 15,1 jours. Trente-huit pour cent ( $\mathrm{n}=9)$ des patientes ont présenté des complications découlant de l'insertion de la sonde, et trois d'entre elles ont été traitées pour infection du lieu d'insertion. Dans cinq cas, des fuites se sont produites autour du site d'insertion, et une des femmes concernées s'est plainte d'inconfort au niveau du site; dans les cinq cas, la situation a été gérée par l'application d'une crème protectrice. Une des patientes a ressenti des douleurs extrêmes associées à un pneumopéritoine découlant de l'insertion de la sonde. On a traité la douleur au moyen d'analgésiques, et le pneumopéritoine s'est résorbé en moins de 48 heures.

Lors de l'obtention de leur congé, $79 \%$ des patientes $(\mathrm{n}=19)$ prenaient simultanément divers médicaments pour la gestion des symptômes associés à l'occlusion intestinale et à l'évolution du cancer (p. ex. antiémétiques, agents stimulant la motilité gastrique, analgésiques et antagonistes des récepteurs $\mathrm{H} 2$ ). Lors de leur congé, on a remis à vingtneuf pour cent des patientes $(n=7)$ un dispositif de traitement de réhydratation parentérale, fonctionnant soit par hypodermoclyse soit par accès veineux central. La réhydratation avait lieu au cours de la nuit ou de façon continue, dépendamment de l'absorption orale de la patiente et de ses préférences personnelles. Au congé, sept des patientes recevaient un traitement de laxatifs ou de lavements. Par la suite, quatre des patientes $(17 \%)$ ont subi des traitements de chimiothérapie palliative de façon à réduire la carcinomatose intestinale et à soulager les symptômes de l'occlusion. Une des patientes n'avait jamais reçu de traitement de chimiothérapie; sa chimiothérapie a eu lieu après l'insertion de la sonde et le retour des fonctions intestinales. La patiente était encore en vie 364 jours après la pose de la sonde. Trois des patientes ont reçu des traitements de chimiothérapie visant à restaurer les fonctions intestinales et à prolonger la durée de survie. Deux patientes ont dû mettre fin à leur chimiothérapie pour des raisons de toxicité, tandis qu'une autre a complété six cycles de Topotecan et a survécu pendant 274 jours après l'insertion de la sonde. La chimiothérapie a probablement prolongé sa vie, mais elle n'a jamais pu tolérer un régime d'aliments solides, et elle a passé $20 \%$ de son temps à l'hôpital afin de contrôler les effets toxiques liés à la chimiothérapie et de traiter les symptômes chroniques de l'occlusion. Elle a toutefois vécu assez longtemps pour assister à la naissance de son premier petit-enfant.

Lors de leur congé, $75 \%$ des patientes ne souffraient plus de nausée et $88 \%$ d'entre elles ne vomissaient plus; $17 \%$ d'entre elles se plaignaient de crampes abdominales et $17 \%$, de ballonnement abdominal. Lors de leur congé, $92 \%$ des patientes ont pu recommencer à recevoir une alimentation par voie orale. Vingt-neuf pour cent d'entre elles pouvaient supporter les liquides clairs, $42 \%$ sont passées à un régime incluant tous les liquides et $21 \%$ avaient un régime qui alternait entre les liquides et les aliments à résidu faible.

Dans $33 \%$ des cas, le drainage de la sonde de GEP se faisait par gravité, tandis que dans $17 \%$ des cas, les sondes étaient reliées à un dispositif d'aspiration, en raison des vomissements persistants lors du drainage par gravité. Cependant, dans les autres $50 \%$ des cas, aucune donnée n'était disponible sur le blocage de la sonde ni sur le drainage par gravité ou par aspiration.

Vingt patientes $(83 \%)$ ont obtenu leur congé de la clinique de soins intensifs. Cinquante-huit pour cent des patientes ont été retournées à domicile avec le suivi des professionnels de la santé. Les soins médicaux à domicile étaient assurés par un médecin qui était disponible pour faire des visites à domicile. Les soins infirmiers palliatifs, qui incluaient le travail par quarts, étaient fournis selon les besoins des patientes et selon les disponibilités. Vingt-cinq pour cent des patientes ont été aiguillées vers un hôpital. Treize pour cent des patientes sont décédées à l'hôpital dans les vingt-six jours suivant leur admission pour cause d'occlusion intestinale, et une patiente a été transférée dans un hôpital communautaire situé près de chez elle.

Des vingt patientes ayant reçu leur congé du contexte de soins intensifs, six (30\%) ont dû être réadmises après l'insertion de la sonde de GEP. En tout, on a compté vingt réadmissions; trois des patientes ont été réadmises plus d'une fois. Deux des réadmissions ont eu lieu pour raison de complications liées à la sonde; dix pour gérer les symptômes de l'occlusion; finalement, on a compté quatre réadmissions pour des raisons qui n'étaient reliées ni à la sonde ni à l'occlusion intestinale. La première réadmission a eu lieu en moyenne 21,7 jours après que la patiente avait obtenu son congé (pour une étendue de 5 à 60 jours). Après l'insertion de la sonde de GEP, la durée médiane de survie des patientes était de 42 jours (de 5 à 1226 jours pour l'ensemble du groupe).

\section{Discussion}

L'occlusion intestinale, surtout celle de l'intestin grêle, est une affection courante chez les femmes atteintes d'un cancer de l'ovaire récurrent ou progressif (Gadducci et coll., 1998). Un des objectifs premiers de la gestion de l'occlusion intestinale lors de la récurrence de la maladie, est le contrôle des symptômes et la palliation. Cette étude a montré que l'insertion d'une sonde de GEP contribue au contrôle de tous les symptômes - sauf la nausée - chez $75 \%$ des patientes. Le degré d'intensité de la nausée et son impact sur le bien-être général de la patiente ne pouvaient être établis à partir des dossiers. Dans l'ensemble, 65 \% des patientes n'avaient pas de symptômes lors de leur congé. Quatre-vingt-douze pour cent des patientes sont retournées à une alimentation par voie orale, $83 \%$ ont reçu leur congé de la clinique de soins intensifs, et $70 \%$ n'ont pas dû être réadmises.

Il n'existe pas beaucoup d'études portant sur les sondes de GEP et leur incidence sur le contrôle des symptômes de l'occlusion. Les résultats disponibles se limitent souvent à la survie après l'insertion de la sonde et aux complications liées à l'insertion. Cette étude fournit des renseignements qui pourraient s'avérer utiles pour les cliniciens et cliniciennes et qui pourraient servir de point de départ pour d'autres recherches.

On aurait pu améliorer le contrôle des symptômes chez les patientes en optimisant l'administration de quelques-uns de leurs médicaments concomitants. Bien que le traitement pharmacologique de chaque patiente était similaire, nous avons prescrit des doses moindres que celles recommandées dans la littérature (Baumrucker, 1998; Gadducci et coll., 1998; Mangili et coll., 1996; Ripomonti, 1994). La plupart des études sur la gestion pharmacologique de l'occlusion intestinale ont été réalisées au sein d'hôpitaux. Chaque patiente était alors suivie de façon constante, et les professionnels de la santé avaient accès aux connaissances spécialisées en matière de soins palliatifs et pouvaient modifier le traitement pharmacologique selon les besoins de chaque patiente quant aux types et aux doses de médicaments. Il se peut que ce genre d'approche soit également efficace en milieu hospitalier.

Les femmes atteintes du cancer de l'ovaire préfèrent souvent rester à domicile parce que, dans bien des cas, elles ont encore une bonne capacité fonctionnelle lorsque survient l'occlusion. Mais il faut que leurs symptômes soient bien gérés. Il se peut que la sonde de GEP constitue une option intéressante pour maîtriser les symptômes de l'occlusion intestinale maligne chez ces femmes. Dans de nombreux cas, ces patientes et leur famille manifestent la crainte que la patiente meure de déshydratation et de sous-alimentation. La capacité de retourner à une alimentation par voie orale est souvent perçue comme un signe de réussite en matière de contrôle des symptômes de l'occlusion, et, dans le cadre de cette étude, le taux de retour à une alimentation par voie orale était élevé. Ces résultats suggèrent que la sonde de GEP aide possiblement les patientes à rester chez elles, puisqu'elle contribue à assurer l'administration d'une alimentation par voie orale pendant la phase de gestion des symptômes. Toutefois, nous n'avons pas pu établir quelle quantité d'alimentation était absorbée au niveau de l'intestin ni quelle quantité s'échappait par la sonde de GEP. L'absence de vomissements est également un important indicateur de contrôle des symptômes de l'occlusion. Ce critère s'avérait significatif 
dans le cadre de notre étude, puisque plus des deux tiers des patientes n'ont pas vomi après l'insertion de la sonde de GEP.

Un peu plus d'un tiers des patientes incluses dans cette étude ont présenté des complications découlant de l'insertion de la sonde de GEP. Il existe peu d'études portant sur les complications telles que les fuites et les infections localisées exigeant l'utilisation d'antibiotiques topiques. Dans une étude, l'érythème et la sensibilité autour du site d'insertion étaient les problèmes les plus souvent rapportés, mais leur incidence n'était pas signalée (Malone, Koone, Larson, Freedman Carrasco et Patton, 1986). Hopkins, Roberts et Morley (1987) rapportent un taux d'infection de $50 \%$ autour du lieu d'insertion, et Marks et collègues (1993) ne rapportent pas de fuites ni d'infections pour lesquelles ils auraient utilisé des antibiotiques prophylactiques. Adelson et Kasowitz (1993) mentionnent un cas (7 \%) de sepsie. Malone et collègues (1986) mentionnent de la douleur chez une patiente $(10 \%)$ qui a exigé l'administration continue d'un analgésique. Une autre patiente $(10 \%)$ a souffert de fièvre pendant 24 heures après l'insertion de la sonde. Il est difficile d'établir si notre taux général de complications est comparable à celui observé dans le cadre d'autres études, mais un taux de $4 \%(\mathrm{n}=1)$ de complications graves est assez peu courant. Il s'agit du seul cas de pneumopéritoine en six ans d'expérience avec les sondes de GEP.

L'échantillon de patientes incluses dans cette étude et qui ont par après reçu des traitements de chimiothérapie est assez réduit. Bien que nous appuyions les conclusions de l'étude d'Abu-Rustum et collègues (1997) à l'effet que les traitements de chimiothérapie devraient être réservés aux patientes n'en ayant jamais reçu, de tels traitements en agent simple continuent d'être administrés aux femmes qui les demandent dans le cadre d'une thérapie agressive visant à contrôler leur cancer récurrent et leur occlusion intestinale. Dans notre établissement, la tendance a été d'offrir des traitements de chimiothérapie au moins deux ou trois semaines après le retour des femmes à domicile, une fois la sonde de GEP insérée. Il est essentiel que ces femmes et leur famille comprennent bien les limites et les risques de la chimiothérapie. De plus, les femmes doivent pouvoir tolérer un régime composé exclusivement de liquides, et avoir une capacité fonctionnelle relativement bonne.

D'autres auteurs (p. ex. Fainsigner et coll., 1994; Gemlo et coll., 1986; Ripamonti, 1994) ont utilisé pour leurs patientes soit la réhydratation par ligne de perfusion centrale, soit l'hypodermoclyse au cours de la nuit ou de façon continue afin de prévenir la déshydratation. Chez $29 \%$ de nos patientes, nous avons utilisé un dispositif de réhydratation afin de prévenir la faiblesse due à la déshydratation ou afin de réconforter les patientes qui craignaient mourir de sous-alimentation et de déshydratation.

\section{Répercussions sur les soins infirmiers}

Les infirmières et infirmiers jouent un rôle fondamental dans le processus d'éducation des patientes et de leur famille, processus qui vise à aider ces dernières à prendre des décisions quant au traitement de l'occlusion intestinale. Confrontées à cette affection, les patientes et leur famille ont de très grands besoins d'information au sujet de ses causes, des options de traitement et des soins de soutien disponibles. Il n'y a pas encore de consensus quant à la meilleure forme de gestion des symptômes associés à l'occlusion intestinale, et par conséquent, les infirmières doivent aider les patientes et leur famille à définir leurs objectifs tant à court terme qu'à long terme. Les infirmières doivent également communiquer ces décisions à l'équipe multidisciplinaire de soins afin qu'un programme de traitement soit établi en fonction des objectifs de la patiente et de sa famille. L'occlusion intestinale étant souvent le premier signe d'une maladie récurrente, l'appui et l'information au sujet de la récurrence et du pronostic contribuent à la complexité des soins et méritent donc une attention soutenue de la part des infirmières.

Dans le cadre de la gestion de l'occlusion intestinale au moyen d'une sonde de GEP, les infirmières jouent un rôle important dans l'évaluation régulière des symptômes des patientes. Une part importante des soins infirmiers devra être consacrée à aider la patiente et sa famille à apprendre à s'occuper correctement de la sonde de GEP et à évaluer l'état de la patiente de façon continue afin de déterminer si le drainage doit se faire par gravité ou par aspiration ou si, au contraire, il convient de bloquer la sonde. Dans cette étude, des données sur le drainage (par gravité, par aspiration ou sonde bloquée) n'étaient disponibles que dans $50 \%$ des cas. Par conséquent, il est difficile de tirer des conclusions en fonction de cette seule étude. De plus, la description des symptômes et de la gestion était rapportée de façon peu méthodique dans divers endroits des dossiers. L'optimisation des soins infirmiers et de la communication pluridisciplinaire devra nécessairement passer par l'amélioration de la description des symptômes associés à l'occlusion intestinale tels que la nausée, les vomissements, les fonctions intestinales et la gestion de la sonde de GEP. Ceci facilitera la personnalisation des interventions, y compris les médicaments concomitants utilisés en tant qu'adjuvants de la sonde de GEP. Par conséquent, nous avons dégagé le besoin d'élaborer un outil qui aidera les infirmières à évaluer l'état des patientes et à mettre en oeuvre les interventions nécessaires. Nous prévoyons mettre sur pied un groupe de travail qui aura pour mandat de déterminer l'outil le plus adéquat, p. ex. plans de soins standard, directives médicales, schémas de traitement ou lignes directrices relatives aux interventions.

La période moyenne du séjour des patientes impliquées dans notre étude était de 15,1 jours. Il convient d'effectuer d'autres études afin de déterminer si la longueur du séjour pourrait être raccourcie en améliorant la planification des congés tout en tenant compte du fait que les répercussions de l'occlusion intestinale peuvent être trop accablantes pour les patientes et leur famille. Ceci étant dit, la période moyenne entre l'admission et l'insertion de la sonde de GEP était de 8,1 jours, et $50 \%$ des patientes incluses dans notre étude avaient déjà été admises une ou deux fois auparavant pour raison d'occlusion intestinale. Il conviendrait donc de commencer à renseigner les patientes et leur famille au sujet de la sonde dès la première admission pour raison d'occlusion, même dans les cas où l'affection de la patiente semble se résorber spontanément, puisque les études démontrent que dans 32 à $45 \%$ des cas, les patientes subiront une réocclusion (Feuer et coll., 2000).

\section{Conclusion}

Les infirmières en oncologie qui soignent les femmes atteintes d'un cancer de l'ovaire et leur famille doivent posséder une solide compréhension de l'occlusion intestinale maligne, des options de traitement et des limites de ces dernières. Cette étude d'amélioration de la qualité indique que la sonde de GEP peut contribuer au contrôle des symptômes de l'occlusion intestinale chez les patientes atteintes d'un cancer récurrent de l'ovaire et que cette sonde a permis d'améliorer les soins prodigués aux patientes de notre établissement.

Il convient d'effectuer de nouvelles études afin de mieux comprendre l'impact de l'occlusion intestinale sur les femmes atteintes d'un cancer de l'ovaire, et d'identifier la meilleure façon de gérer les symptômes de l'occlusion intestinale découlant de ce cancer. Ces travaux de recherche doivent inclure des mesures de la qualité de vie, et les mesures des résultats doivent être normalisées. Dans leur examen des données provenant de la base de données Cochrane, Feuer et coll. (2000) recommandaient que l'on utilise les mesures relatives à la nausée, aux vomissements, à la douleur et à la constipation en tant qu'indicateurs de l'issue de la chirurgie pour occlusion intestinale. Il convient d'employer les mêmes indicateurs pour tous les types de traitement, et non pas seulement dans le cas de la sonde de GEP, cela afin de déterminer les meilleures options disponibles. D'autres questions de recherche qu'il importe d'aborder sont les suivantes: Du point de vue physique et psychosocial, quel est l'impact de l'hydratation sur le contrôle des symptômes dans le cadre des soins palliatifs pour l'obstruction intestinale? En tant que stratégie de soins palliatifs, la chimiothérapie présente-t-elle des avantages pour les patients qui souhaitent vivre le plus longtemps possible? 
Abu-Rustum, N., Barakat, R.R., Venkatramann, E., \& Spriggs, D. (1997). Chemotherapy and total parenteral nutrition for advanced ovarian cancer with bowel obstruction. Gynecologic Oncology, 64, 493-495.

Adelson, M.D., \& Kasowitz, M.H. (1993). Percutaneous endoscopic drainage gastrostomy in the treatment of gastrointestinal obstruction from intraperitoneal malignancy. Obstetrics \& Gynecology, 81(3), 467-471.

Baumrucker, S. (1998). Management of intestinal obstruction in hospice care. The American Journal of Hospice \& Palliative Care, 232-235.

Beattie, G., Leonard, R.C.F., \& Smyth, J.F. (1989). Bowel obstruction in ovarian carcinoma: A retrospective study and review of the literature. Palliative Medicine, 3, 275-280.

Cain, J., Wenzel, L., Monk, B., \& Cella, D. (1998). Palliative care and quality of life. In D.M. Gershenson \& W.P. McGuire (Eds.), Ovarian cancer: Controversies in management (pp. 281-303). Edinburgh, Scotland: Churchill Livingston.

DiScia, P.J., \& Creasman, W.T. (2002). Epithelial ovarian cancer. Clinical gynecologic oncology (pp. 289-343). St. Louis, MO: Mosby.

Fainsinger, R.L., Spachynski, K., Hanson, J., \& Bruera, E. (1994). Symptom control in terminally ill patients with malignant bowel obstruction. Journal of Pain and Symptom Management, 9(1), 12-18.

Feuer, D.J., Broadley, K.E., \& Barton, D.P.J. (2000). Surgery for the resolution in malignant bowel obstruction in advanced gynecological and gastrointestinal cancer. Cochrane Database of Systematic Reviews, 4, CD002764.

Frankel Kelvin, J., \& Scagliola, J. (1998). Metastases involving the gastrointestinal system. Seminars in Oncology Nursing, 14(3), 187-198.

Gadducci, A., Iacconi, I., Fanucchi, A., Cosio, S., Miccoli, P., \& Genazzani, A.R. (1998). Survival after intestinal obstruction in patients with fatal ovarian cancer: Analysis of prognostic variables. International Journal of Gynecological Cancer, 8, 177-182.

Gemlo, B., Rayne, A.A., Lewis, B., Wong, A., Viele, C.S., Ungaretti, J.R., et al. (1986). Home support of patients with end-stage malignant bowel obstruction using hydration and venting gastrostomy. The American Journal of Surgery, 152, 100-104.
Hopkins, M.P., Roberts, J.A., \& Morley, G.W. (1987). Outpatient management of small bowel obstruction in terminal ovarian cancer. The Journal of Reproductive Medicine, 32(11), 827-829.

Jong, P., Sturgeon, J., \& Jamieson, C.G. (1995). Benefit of palliative surgery for bowel obstruction in advanced ovarian cancer. Canadian Journal of Surgery, 5, 454-457.

Kehoe, S., \& Mann, C. (2002). Role of surgery in relapse and palliation of ovarian cancer. In I. Jacobs, J. Shepherd, D. Oram, A. Blackett, D. Luesley, A. Berchuck, \& C. Hudson (Eds.), Ovarian cancer, (pp. 305-309). Oxford, England: Oxford University Press.

Khoo, D., Hall, E., Motson, R., Riley, J., Denman, K., \& Waxman, J. (1994). Palliation of malignant intestinal obstruction using Octriotide. European Journal of Cancer, 30A, 28-30.

Malone, J.M., Koonce, T., Larson, D.M., Freedman, R.S., Carrasco, C.H.O., \& Saul, P.B. (1986). Palliation of small bowel obstruction by percutaneous gastrostomy in patients with progressive ovarian carcinoma. Obstetrics \& Gynecology, 68(3), 431-433.

Mangili, G., Franchi, M., Mariani, A., Zanaboni, F., Rabaitti, E., Frigerio, L., et al. (1996). Octriotide in the management of bowel obstruction in terminal ovarian cancer. Gynecologic Oncology, 61, 345-348.

Marks, W., Perkal, M.F., \& Schwartz, P.E. (1993). Percutaneous endoscopic gastrostomy for gastric decompression in metastatic gynecologic malignancies. Surgery, Gynecology and Obstetrics, 177, 573-576.

Mercandante, S., Spoldi, E., Caraceni, A., Maddaloni, S., \& Simonetti, M.T. (1993). Octriotide in relieving gastrointestinal symptoms due to bowel obstruction. Palliative Medicine, 7, 295-299.

National Cancer Institute of Canada. (2002). Canadian cancer statistics 2002. Toronto: Author.

Ripomonti, C. (1994). Management of bowel obstruction in advanced cancer. Current Opinion in Oncology, 6, 351-357.

van Ooijen, B., van der Burg, M.E.L., Planting, A.S.Th., Siersema, P.D., \& Wiggers, T. (1993). Surgical treatment or gastric drainage only for intestinal obstruction in patients with carcinoma of the ovary or peritoneal carcinomatosis of other origin. Surgery, Gynecology and Obstetrics, 176, 469-474. 